\title{
COMPARISON BETWEEN SINGLE AND DOUBLE INTEGRAL TRANSFORMATION SOLUTIONS OF HEAT CONDUCTION IN SOLID-STATE ELECTRONICS
}

\author{
L. M. Corrêa, \\ and D. J. N. M. Chalhub \\ Universidade do Estado do Rio de Janeiro \\ Departamento de Engenharia Mecânica \\ Group for Studies and Environmental \\ Simulations in Reservoirs (GESAR) \\ Rua Fonseca Teles, 121 \\ CEP. 20940-200, Rio de Janeiro, RJ, Brasil \\ livcorrea@yahoo.com.br \\ daniel.chalhub@uerj.br \\ Received: September 20, 2018 \\ Revised: October 11, 2018 \\ Accepted: November 01, 2018
}

\section{ABSTRACT}

The design of modern electronic devices has been dealing with challenges on thermal control. In this work, it is proposed two different ways of modeling the temperature field in Solid State Electronics (SSE) using integral transforms, with several heat generations in the domain of the microchip and external convection. Two proposed approaches solve the heat conduction formulation on the SSE using the Classical Integral Transform Technique (CITT): One performing a single transformation (CITT-ST) and the other performing a double transformation (CITT-DT). Both methodologies are compared and achieved similar results. The simpler analytical solution by CITT-DT contrasts with a complex and cumbersome analytical manipulation of CITT-ST. The results show that CITT-ST is more efficient to obtain the solution, requiring a lower truncation order, for the problem of heat conduction in Solid State Electronics even though it has a more complex formulation.

Keywords: thermal control; solid state electronics; multiple heat generation; classical integral transform technique

\section{NOMENCLATURE}

$\mathrm{T} \quad$ Temperature, ${ }^{\circ} \mathrm{C}$

$\mathrm{k}$ Thermal conductivity, $\mathrm{W} /(\mathrm{m} . \mathrm{K})$

$\mathrm{L} \quad \mathrm{x}$-dimension of the SSE, $\mathrm{m}$

$\mathrm{H} \quad \mathrm{y}$-dimension of the SSE, $\mathrm{m}$

$T_{f} \quad$ Temperature of the surrounding air, ${ }^{\circ} \mathrm{C}$

$T_{0} \quad$ Reference temperature, ${ }^{\circ} \mathrm{C}$

$h \quad$ Convection heat transfer coefficient, $\mathrm{W} /\left(\mathrm{m}^{2} . \mathrm{K}\right)$

Bi Biot Number

N Norm

$\dot{q}_{c}$ " Convection heat flux, $\mathrm{W} / \mathrm{m}^{2}$

$\dot{q}_{x} "$ Heat flux in $\mathrm{x}$ direction, $\mathrm{W} / \mathrm{m}^{2}$

$\dot{q}_{y} " \quad$ Heat flux in y direction, $\mathrm{W} / \mathrm{m}^{2}$

$\dot{g}^{\prime \prime} \quad$ Heat generation, $\mathrm{W} / \mathrm{m}^{3}$

$G \quad$ Dimensionless Heat Generation (HG)

$G_{e} \quad$ Total HG intensity of each internal component

$\dot{E} \quad$ Energy rate

$x, y$ Cartesian coordinates

\section{Greek symbols}

$\xi, \eta \quad$ Dimensionless cartesian coordinates

$\delta \quad$ z-dimension of the SSE, m

$\theta \quad$ Dimensionless temperature

$\beta \quad$ Aspect ratio between $\mathrm{L}$ and $\mathrm{H}$

$\gamma \quad$ Aspect ratio between $\mathrm{L}$ and $\delta$

$\bar{\theta} \quad$ Transformed dimensionless temperature

$\overline{\bar{\theta}} \quad$ Double transformation dimensionless temperature

\section{$\lambda, \mu$ Eigenvalues \\ $\Psi, \Xi$ Eigenfunctions}

\section{Subscripts}

$n, m$ Integral transform index

$n_{\max }$ truncation order for CITT

$k$ Index of the HG of each internal component

$k_{\max }$ Number of HG internal component

\section{INTRODUCTION}

Solid-State Electronics (SSE) or devices are circuits built entirely from solid materials and in which the electrons or other charge carriers, are confined entirely within the solid material. They were responsible for the electronical revolution, marked by the Nobel prize in 1956 for the transistor invention, (Chelikowsky and Franciosi, 1991). The decrease of the size of integrated circuits and the need for a better and efficient power dissipation has motivated several studies about the temperature control. Thermal control of electronic components focusses on keeping the components' temperature equal to or below the manufacturer's maximum specified service temperature, typically between 85 and $100^{\circ} \mathrm{C}$, (Peterson and Ortega, 1994). The knowledge of those values and their ideal operational ranges would ensure an effective performance and long service life; in contrast, a violation of which, can significantly compromise the reliability of the device, (Iyengar and Schmidt, 2006). 
The thermal management in SSEs has motived several works aiming to understand the thermal effects in electronic devices. Abdelmlek et al. (2016), for instance, have evaluated the effect of thermal conduction on Light Emitting Diodes (LED), since it doesn't irradiate heat but increase the junction temperature on its interior. More recently, Xiao et al. (2017) investigated the thermal performance of 3D stacked integrated circuits based on heat transfer theory under natural convection environment.

The hot spot effect is the temperature increase in specific regions on the chip where the heat flux is higher than in the chip average. Hot spots temperatures are often from $10^{\circ} \mathrm{C}$ to $25^{\circ} \mathrm{C}$ higher than average, (Iyengar and Schmidt, 2006). Bar-Cohen and Wang (2009) described on chip thermal management approaches for hot spots remediation.

Analytical methods have been improved to obtain an exact solution for proposed electronic problems. One example is the work of Rinaldi (2000), which presented an analytical model of thermal distribution in ICs based on a classic method of electrostatics. Rinaldi also shows the effect of the geometry and layout on thermal behavior.

The integral transform technique is an analytical method which provides a systematic, efficient, and straightforward approach for the solution of both homogeneous and nonhomogeneous, steady state, and time-dependent boundary value problems of heat conduction. It is presented by Cotta (1993), Hahn and Özisik (2012) and the technique has been applied by several authors. Sphaier and Cotta (2000) applied the Integral Transform Technique on the solution of a multidimensional partial differential models within irregularly shaped domains. Braga Junior and Sphaier (2014) has applied the General Integral Transform Technique (GITT) for the solution of heat transfer in Functionally Graded Materials (FGM), with different thermal conductivity variation in the material. Chalhub et al. (2014) proposed a solution for the Poisson equation arising from the incompressible Navier-Stokes equations by two methodologies using the Classic Integral Transform Technique (CITT): a single transformation and a double transformation. Although both techniques presented a very similar convergence behavior and results, the double transformation has poorer performance in comparison with the single transformation scheme. Even though the performance of the double transformation was not so good, this formulation has a simpler analytical solution, which might be more interesting in some cases such as the problem that was presented. Dantas (1996) applied Integral Transforms for solving a heat transfer problem in microchips with plastic encapsulation and different layouts of thermal conductivity. For this problem, the thermal conductivity is considered constant and is under natural convection effects. Furthermore, the research developed by Silva et al. (2017) presented a formulation for the solution of the two-dimensional steady state heat conduction with heat generation on a plate. The CITT was used to solve the problem in a semi-analytical manner and a second order central finite difference method (FDM) was also implemented, the convergence analysis showed that CITT has a greater performance having no difficulties to obtain accurate results with very few terms in the solution summation while FDM struggled specially for the positions near the center and for high concentration of heat generation in the center of the plate.

The present work proposes two different versions of the analytical approach utilizing the Classical Integral Transform Technique (CITT) to obtain the solution of heat conduction in a Solid-State Electronic (SSE) with several internal components, each of which is a source of Heat Generation (HG). It is considered an SSE with heat convection on the top of surface of the substrate. On the bottom of the SSE, the surface is in contact with the board, hence the boundary condition considered is insulation. Since the thickness of the device is small compared to other dimensions, a partial lumping approach in z-direction is performed and the final mathematical formulation is two dimensional. The heat generation is composed by several generation in piecewise constant function. The comparison between CITT single and double transformations in solid state electronics is described considering the number of requiring terms to obtain the final converged value.

\section{PROBLEM FORMULATION}

The mathematical formulation of the heat conduction in an electronic microchip is given by the energy equation in steady-state after applying a partial lumping approach in z-direction. For this work, it is assumed the heat conductivity as constant inside the microchip.

The schematic illustration of SSE and the infinitesimal volume control are shown on the figure 1 and the equation of energy conservation is written considering the heat fluxes, explained on table 1.

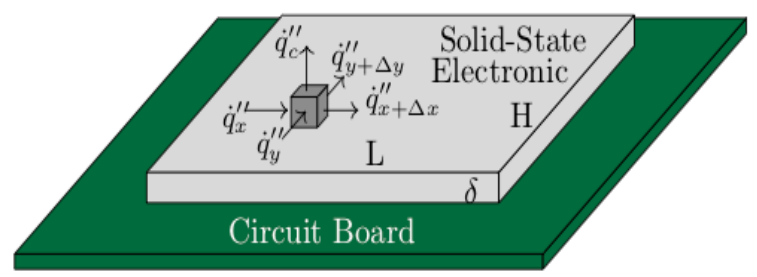

Figure 1. Heat conduction in the SSE.

The SSE top surface is affected by the heat convection with the surrounding air, hence the boundary condition is heat flux by convection ( $\dot{q}_{c} "$ ). On the other hand, the bottom surface is in contact with the circuit board, hence, it is considered insulated $\left(\dot{q}^{\prime \prime}=0\right)$. The heat generation $\left(\dot{g}^{\prime \prime}\right)$ is also 
considered for this formulation.

The formulation of the problem is shown below with its respective boundary conditions, for $0 \leq x \leq L$ and $0 \leq y \leq H$.

$$
\begin{gathered}
k\left(\frac{\partial^{2} T}{\partial x^{2}}+\frac{\partial^{2} T}{\partial y^{2}}\right)+\dot{g}^{\prime \prime}(x, y)=\frac{h\left(T-T_{f}\right)}{\delta} \\
\left.\frac{\partial T}{\partial x}\right|_{x=0}=0 ;\left.\frac{\partial T}{\partial y}\right|_{y=0}=0 \\
\left.\frac{\partial T}{\partial x}\right|_{x=L}=0 ;\left.\frac{\partial T}{\partial y}\right|_{y=H}=0 ;
\end{gathered}
$$

where $\mathrm{T}$ is the temperature, $\mathrm{k}$ is the thermal conductivity, $\dot{g}^{\prime \prime}$ is the heat generation, $T_{f}$ is the environment air temperature, $h$ is the convection heat transfer coefficient and $\mathrm{L}, \mathrm{H}$ and $\delta$ are the dimensions of the chip in $\mathrm{x}, \mathrm{y}$ and $\mathrm{z}$ directions respectively.

The nondimensionalization of the problem leads to the following mathematical formulation:

$$
\begin{gathered}
\left(\frac{\partial^{2} \theta}{\partial \xi^{2}}+\beta^{2} \frac{\partial^{2} \theta}{\partial \eta^{2}}\right)-B i_{L} \gamma \theta=-G(\xi, \eta) \\
\left.\frac{\partial \theta}{\partial \xi}\right|_{\xi=0}=0 ;\left.\quad \frac{\partial \theta}{\partial \eta}\right|_{\eta=0}=0 ; \\
\left.\frac{\partial \theta}{\partial \xi}\right|_{\xi=1}=0 ;\left.\quad \frac{\partial \theta}{\partial \eta}\right|_{\eta=1}=0 ;
\end{gathered}
$$

The non-dimensional groups are defined as:

$$
\begin{gathered}
\xi=\frac{x}{L} ; \quad \eta=\frac{y}{H} \\
\theta=\frac{T-T_{f}}{T_{0}-T_{f}} ; \quad B i=\frac{h L}{k} \\
\beta=\frac{L}{H} ; \quad \gamma=\frac{L}{\delta} ; \quad G(\xi, \eta)=\frac{\dot{g}^{\prime \prime \prime} L^{2}}{k \Delta T}
\end{gathered}
$$

where $\beta$ and $\gamma$ are aspect ratios, $B i_{L}$ is the Biot number, $\theta$ is the dimensionless temperature, $\xi$ and $\eta$ are the dimensionless versions of $x$ and $y$; and $G$ is composed by several generation in piecewise constant function over the domain of the chip.

\section{SUPERPOSITION METHOD}

The Superposition Method is introduced by Hahn and $\mathrm{O} \square$ zisik (2012) for solving nonhomogeneous linear problems. Since this particular problem is linear, this method can be applied and the equation (4) is divided in each individual heat generation as follows:

$$
\frac{\partial^{2} \theta_{k}}{\partial \xi^{2}}+\beta^{2} \frac{\partial^{2} \theta_{k}}{\partial \eta^{2}}-B i \gamma \theta_{k}=-G_{k}
$$

where $k$ represents each internal component that generates heat.

The final temperature is obtained by summing all partial temperatures:

$$
\theta=\sum_{k=1}^{k_{\max }} \theta_{k}
$$

In order to solve the proposed problem (10) for each internal heat generation $k$, the Classical Integral Transform Technique (CITT) is applied.

\section{SOLUTION BY CLASSICAL INTEGRAL TRANSFORM TECHNIQUE - SINGLE TRANSFORMATION (CITT-ST)}

The CITT is an analytical technique that uses expansions of the sought solution in terms of an infinite orthogonal basis of eigenfunctions, keeping the solution process always within a continuous domain. To establish the transformation pair, the temperature field is written as function of an orthogonal eigenfunctions obtained from the following auxiliary eigenvalue problem known as the Helmholtz classic problem in cartesian coordinates, where $\Psi(\eta)$ are the eigenfunctions and $\lambda_{n}$ are the eigenvalues. For this particular problem, the case where $\lambda=0$ also exists.

$$
\begin{aligned}
& \Psi_{n}^{\prime \prime}(\eta)+\lambda_{n}^{2} \Psi_{n}(\eta)=0 \\
& \Psi_{n}^{\prime}(0)=0 ; \Psi_{n}^{\prime}(1)=0
\end{aligned}
$$

where the solution of the eigenvalue problem is given by, for $\lambda=0$ :

$$
\begin{aligned}
& \Psi_{0}(\eta)=1 \\
& \lambda_{0}=0
\end{aligned}
$$

and for $\lambda>0$ :

$$
\begin{aligned}
& \Psi_{n}(\eta)=\cos \left(\lambda_{n} \eta\right) \\
& \lambda_{n}=n \pi \text { for } n=1,2,3, \ldots
\end{aligned}
$$

To apply the CITT, the transformation pair is defined. 


$$
\begin{gathered}
\text { Transform } \Rightarrow \bar{\theta}_{n}(\xi)=\int_{0}^{1} \theta_{k}(\xi, \eta) \Psi_{n}(\eta) d \eta \\
\text { Inversion } \Rightarrow \theta_{k}(\xi, \eta)=\sum_{n=0}^{\infty} \frac{\bar{\theta}_{n}(\xi) \Psi_{n}(\eta)}{N_{n}}
\end{gathered}
$$

where $\bar{\theta}_{n}$ is the transformed version of $\theta_{k}$ and the norm $N_{n}$ is defined by:

$$
N_{n}=\int_{0}^{1} \Psi_{n}^{2} d \eta
$$

To obtain the transformed equation, both sides of the heat conduction equation (3a) are multiplied by the eigenfunction $\Psi_{n}$ and integrated in the domain. Two transformed equations are obtained: one for $\lambda>0$ and one for $\lambda=0$ :

For $\lambda>0$ :

$$
\begin{aligned}
& \bar{\theta}{ }_{n}-\left(\beta^{2} \lambda_{n}^{2}+B i \gamma\right) \bar{\theta}_{n}=-\bar{G}_{n}(\xi) \\
& \bar{\theta}_{n}^{\prime}(0)=0 ; \quad \bar{\theta}_{n}^{\prime}(1)=0 ;
\end{aligned}
$$

where $\bar{G}_{n}(\xi)$ is given by:

$$
\bar{G}_{n}(\xi)=\int_{0}^{1} G_{k}(\xi, \eta) \Psi_{n}(\eta) d \eta
$$

The transformed equation (18) admits an analytical solution.

For $\lambda=0$ :

$$
\begin{aligned}
& \bar{\theta}{ }_{0}-(B i \gamma) \bar{\theta}_{0}=-\bar{G}_{0}(\xi) \\
& \bar{\theta}_{0}{ }_{0}(0)=0 ; \quad \bar{\theta}_{0}^{\prime}(1)=0 ;
\end{aligned}
$$

where $\bar{G}_{0}$ is given by:

$$
\bar{G}_{0}(\xi)=\int_{0}^{1} G_{k}(\xi, \eta) \Psi_{0}(\eta) d \eta
$$

The transformed equation (20) also admits an analytical solution.

Finally, in order to obtain the partial temperature field $\theta_{k}$, the inversion formula (16) must be utilized and the summation must be truncated to a finite value $\left(n_{\max }\right)$. Moreover, to find the final temperature equation (11) must be used.

\section{SOLUTION BY CLASSICAL INTEGRAL TRANSFORM TECHNIQUE - DOUBLE TRANSFORMATION (CITT-DT)}

The CITT-DT approach performs one transformation for each direction. The double transformation pair is given by:

$$
\begin{gathered}
\text { Transform } \Rightarrow \overline{\bar{\theta}}_{n m}=\int_{0}^{1} \int_{0}^{1} \theta_{k}(\xi, \eta) \Psi_{n}(\eta) \Xi_{m}(\xi) d \eta d \xi \\
\text { Inversion } \Rightarrow \theta_{k}(\xi, \eta)=\sum_{n=0}^{\infty} \sum_{m=0}^{\infty} \frac{\overline{\bar{\theta}}_{n m} \Psi_{n}(\eta) \Xi_{m}(\xi)}{N_{y n} N_{x m}}
\end{gathered}
$$

where $\Psi_{n}(\eta)$ and $\Xi_{m}(\xi)$ are the eigenfunctions. $\overline{\bar{\theta}}_{n m}$ is the transformed version of $\theta_{k} . N_{y n}$ and $N_{x m}$ are the norms and are defined by:

$$
\begin{aligned}
& N_{y n}=\int_{0}^{1} \Psi_{n}^{2} d \eta ; \\
& N_{x m}=\int_{0}^{1} \Xi_{m}^{2} d \xi .
\end{aligned}
$$

The greatest advantage of this approach is that it requires significantly less analytical effort and the final solution is simpler and more compact. The CITT-DT presents, however, the disadvantage of having a double summation, having a poorer convergence performance. The consequence is that the solution requires more terms for its convergence.

The eigenproblem in $\eta$-direction is given by:

$$
\begin{aligned}
& \Psi{ }_{n}(\eta)+\lambda_{n}^{2} \Psi_{n}(\eta)=0 \\
& \Psi_{n}(0)=0 ; \quad \Psi_{n}^{\prime}(1)=0 ; \\
& \Psi_{0}(\eta)=1 \text { for } \lambda_{0}=0 ; \\
& \Psi_{n}(\eta)=\cos \left(\lambda_{n} \eta\right) \text { for } \lambda_{n}=n \pi ; \quad n=1,2,3, \ldots
\end{aligned}
$$

and for $\xi$ - direction:

$$
\begin{aligned}
& \Xi_{m}{ }^{\prime \prime}(\xi)+\mu_{m}^{2} \Xi_{m}^{2}(\xi)=0 \\
& \Xi_{m}^{\prime}(0)=0 ; \quad \Xi_{m}^{\prime}(1)=0 ; \\
& \Xi_{0}(\xi)=1 \text { for } \mu_{0}=0 ; \\
& \Xi_{m}(\xi)=\cos \left(\mu_{m} \xi\right) \text { for } \mu_{m}=m \pi ; \quad m=1,2,3, \ldots
\end{aligned}
$$

Equation (10) is now transformed, multiplying by $\Psi_{n}$ and by $\Xi_{m}$ and integrating in the domain in $\xi$ and $\eta$.

$$
-\mu_{m}^{2} \overline{\bar{\theta}}_{n m}-\beta^{2} \lambda_{n}^{2} \overline{\bar{\theta}}_{n m}-B i \gamma \overline{\bar{\theta}}_{n m}=-\overline{\bar{G}}_{n m}
$$

Solving the equation, it is obtained different solutions for $\theta$ :

For $n \neq 0$ and $m \neq 0$ :

$$
\overline{\bar{\theta}}_{n m}=\frac{\overline{\bar{G}}_{n m}}{\left(\mu_{m}^{2}+\beta^{2} \lambda_{n}^{2}+B i \gamma\right)}
$$

For $n=0$ and $m \neq 0$ :

$$
\overline{\bar{\theta}}_{0 m}=\frac{\overline{\bar{G}}_{0 m}}{\left(\mu_{m}^{2}+B i \gamma\right)}
$$


For $n \neq 0$ and $m=0$ :

$$
\overline{\bar{\theta}}_{n 0}=\frac{\overline{\bar{G}}_{n 0}}{\left(\beta^{2} \lambda_{n}^{2}+B i \gamma\right)}
$$

For $n=0$ and $m=0$ :

$$
\overline{\bar{\theta}}_{00}=\frac{\overline{\bar{G}}_{00}}{B i \gamma}
$$

Equation (27) represents a decoupled algebraic system of equations and is far more simple and compact than the ODE system of the CITT-ST approach and brings the capacity of not having to solve an ordinary differential equation to obtain the solution for $\overline{\bar{\theta}}_{n m}$.

To obtain the final temperature, the inversion formula (23) must be applied together with equation (11). The double summation can be reduced to one as shown in the equation (32) by a reordering scheme, where the higher values of $\overline{\bar{\theta}}_{n m}$ by combinations of $n$ and $m$ are selected first for faster convergence of the series.

$$
\begin{aligned}
& \theta_{k}=\sum_{n=0}^{\infty} \sum_{m=0}^{\infty} \frac{\overline{\bar{\theta}}_{n m}(\xi, \eta) \Psi_{n}(\eta) \Xi_{m}(\xi)}{N_{y n} N_{x m}} \Rightarrow \\
& \theta_{k}=\sum_{q=0}^{\infty} \frac{\overline{\bar{\theta}}_{q}(\xi, \eta) \Psi_{n(q)}(\eta) \Xi_{m(q)}(\xi)}{N_{y n(q)} N_{x m(q)}}
\end{aligned}
$$

where $q$ is reordering scheme new index. The summation must be truncated to a finite value $\left(q_{\max }\right)$.

The transformed heat generations are defined as:

$$
\begin{aligned}
& \overline{\bar{G}}_{n m}=\sum_{k=1}^{k_{\max }} \iint_{\Omega_{k}} G_{k} \Xi_{m} \Psi_{n} d \xi d \eta \\
& \overline{\bar{G}}_{0 m}=\sum_{k=1}^{k_{\max }} \iint_{\Omega_{k}} G_{k} \Xi_{m} \Psi_{0} d \xi d \eta \\
& \overline{\bar{G}}_{n 0}=\sum_{k=1}^{k_{\max }} \iint_{\Omega_{k}} G_{k} \Xi_{0} \Psi_{n} d \xi d \eta \\
& \overline{\bar{G}}_{00}=\sum_{k=1}^{k_{\max }} \iint_{\Omega_{k}} G_{k} \Xi_{0} \Psi_{0} d \xi d \eta
\end{aligned}
$$

where $G_{k}$ represents each heat generation, which are constant values. The domain $\Omega_{k}$ is defined by the area where each component responsible for heat generation on the microchip is located. The total number of generations in the microchip is represented by $k_{\max }$

\section{RESULTS AND DISCUSSION}

After describing the problem formulation and solution methodology, in this section the results are shown. It was selected 0.1 and 0.01 as values for $B i \gamma$ and 1 and 0.8 as values for $\beta$. Each combination of $\mathrm{Bi} \gamma$ and $\beta$ admitted a different solution.

The SSE layout analyzed in this work is shown in figure 2. In this image it is shown that there are five internal components inside the SSE which compose a symmetrical design for the chip and generate heat. The positions of each component are indicated in table $1 . \mathrm{G}_{1}$, for instance, is located between positions 0.4 and 0.6 for both $\xi$ and $\eta$. The component indicated by $k=5$, is located on the lower right region of the chip, between $\xi=0.7$ and $\xi=0.8$ and between $\eta=0.2$ and $\eta=0.3$.

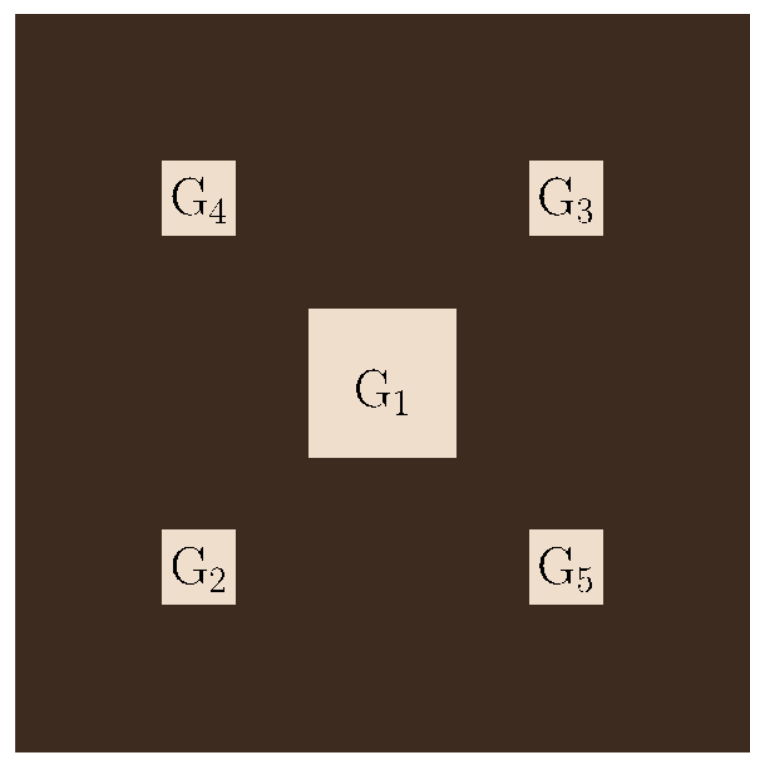

Figure 2. Layout of the heat generation in the SSE.

Table 1. Internal component parameters of the first layout.

\begin{tabular}{|c|c|c|c|c|c|}
\hline$k$ & $\xi_{i}$ & $\xi_{f}$ & $\eta_{i}$ & $\eta_{f}$ & $G_{k}$ \\
\hline 1 & 0.4 & 0.6 & 0.4 & 0.6 & 0.11 \\
\hline 2 & 0.2 & 0.3 & 0.2 & 0.3 & 0.12 \\
\hline 3 & 0.7 & 0.8 & 0.7 & 0.8 & 0.10 \\
\hline 4 & 0.2 & 0.3 & 0.7 & 0.8 & 0.03 \\
\hline 5 & 0.7 & 0.8 & 0.2 & 0.3 & 0.20 \\
\hline
\end{tabular}

Even though the symmetrical design of components exists, each component dissipate heat at different intensities. Consequently, the SSE will present an asymmetrical thermal profile. Each intensity of heat generation of the components is described in the last column of table 1 . The 
component $\mathrm{G}_{5}$ presents the highest intensity, which is 0.20 , and $\mathrm{G}_{4}$ presents the lowest intensity, 0.03 .

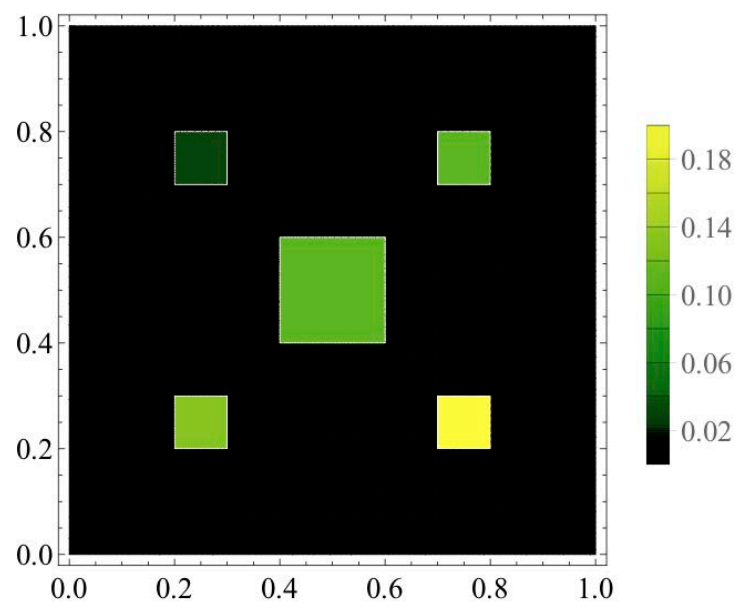

Figure 3. Heat generation distribution in the SSE.

Figure 3 shows the generation values of each component of the SSE. The yellow color indicates the high intensity of G5 and lower intensities are indicated by the green color. The dark green is attributed to G4 due to its lower heat generation. The dark color indicates the absence of internal components, in other words, no heat is generated in this region.

Figure 4 presents the thermal analysis of the SSE. The solution of the problem is shown for $\mathrm{Bi} \gamma=$ 0.01 and $\beta=1$. This thermal profile was achieved after the convergence of CITT-ST. Since CITT-ST and CITT-DT converge to the same final values, figure 4 also represents the results obtained by CITTDT after the convergence.

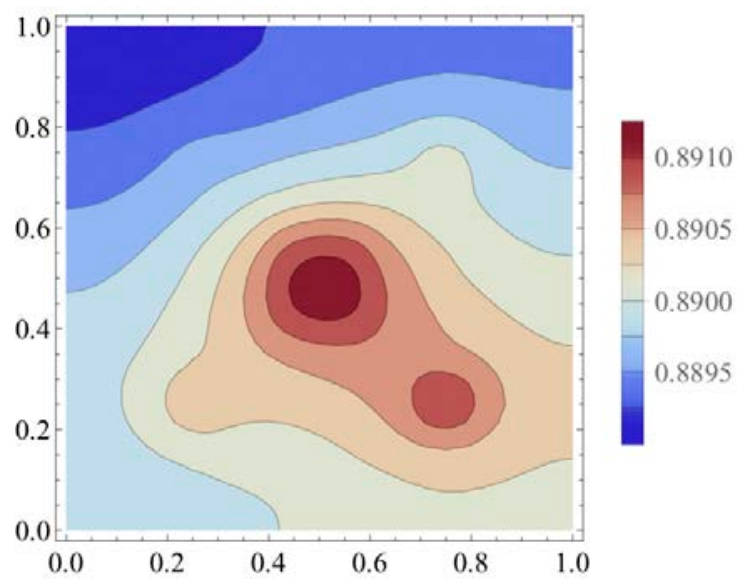

Figure 4. Converged CITT-ST solution for Bi $\gamma=$ 0.01 and $\beta=1$ in the SSE.

The figure 4 exposes the effect of a symmetrical layout but different intensity of its components generates an asymmetrical thermal profile, as was mentioned before. Even though $G_{1}$ has a lower intensity than $G_{5}$, its larger area causes a higher heating where this component $G_{1}$ is located. The reason for it is that heat generations depends on its volume, and in this case because of partial lumping on z-direction, depends on the components' area. $G_{5}$ presents the highest heat generation intensity in this SSE, which can be noticed when comparing its thermal effect with the other same sized components, such as $G_{2}, G_{3}$ and $G_{4}$.

The results of convergence of CITT-DT are presented on table 2 .

Table 2. Temperature $\theta(\xi, \eta)$ Convergence solved by CITT- Double Transformation.

\begin{tabular}{|c|c|c|c|c|}
\hline$n_{\max }$ & $(0.2,0.2)$ & $(0.5,0.5)$ & $(0.6,0.8)$ & $(0.8,0.3)$ \\
\hline \multicolumn{5}{|c|}{ Bi $\gamma=0.1$ and $\beta=1$} \\
\hline 200 & 0.0890455 & 0.0898975 & 0.0888696 & 0.0895100 \\
\hline 250 & 0.0890452 & 0.0898990 & 0.0888696 & 0.0895106 \\
\hline 500 & 0.0890453 & 0.0898981 & 0.0888694 & 0.0895114 \\
\hline 1000 & 0.0890453 & 0.0898982 & 0.0888694 & 0.0895109 \\
\hline 1500 & 0.0890453 & 0.0898982 & 0.0888695 & 0.0895109 \\
\hline \multicolumn{5}{|c|}{ Bi $\gamma=0.01$ and $\beta=1$} \\
\hline 200 & 0.890045 & 0.890898 & 0.889868 & 0.890513 \\
\hline 250 & 0.890045 & 0.890900 & 0.889868 & 0.890513 \\
\hline 500 & 0.890045 & 0.890899 & 0.889868 & 0.890514 \\
\hline 500 & 0.890045 & 0.890899 & 0.889868 & 0.890514 \\
\hline 1000 & 0.890045 & 0.890899 & 0.889868 & 0.890514 \\
\hline 1500 & 0.890045 & 0.890899 & 0.889868 & 0.890514 \\
\hline \multicolumn{5}{|c|}{ Bi $\gamma=0.1$ and $\beta=0.8$} \\
\hline 200 & 0.0891611 & 0.0901247 & 0.0886954 & 0.0896848 \\
\hline 250 & 0.0891603 & 0.0901262 & 0.0886957 & 0.0896854 \\
\hline 500 & 0.0891609 & 0.0901256 & 0.0886956 & 0.0896858 \\
\hline 1000 & 0.0891609 & 0.0901257 & 0.0886955 & 0.0896856 \\
\hline 1500 & 0.0891609 & 0.0901257 & 0.0886955 & 0.0896856 \\
\hline \multicolumn{5}{|c|}{ Bi $\gamma=0.01$ and $\beta=0.8$} \\
\hline 200 & 0.890163 & 0.891126 & 0.889691 & 0.890690 \\
\hline 250 & 0.890163 & 0.891128 & 0.889691 & 0.890610 \\
\hline 500 & 0.890164 & 0.891128 & 0.889691 & 0.890691 \\
\hline 1000 & 0.890164 & 0.891128 & 0.889691 & 0.890691 \\
\hline 1500 & 0.890164 & 0.891128 & 0.889691 & 0.890691 \\
\hline
\end{tabular}

Analyzing table 2, it can be noticed that the lower Bi $\gamma$ favored a faster convergence of the solution, as can be seen for Bi $\gamma=0.01$ where all the selected points have converged within 500 terms. In other cases, such as $\mathrm{Bi} \gamma=0.1$ and $\beta=1$ required 1500 terms for the six-digits convergence at position $(0.6,0.8)$. Although the solution using the CITT-DT is simpler than the CITT-ST, the solution required a higher truncation order of the sum for its convergence.

The results of convergence of CITT-ST are presented on table 3 .

Table 3. Temperature $\theta(\xi, \eta)$ Convergence solved by CITT - Single Transformation.

\begin{tabular}{|c|c|c|c|c|}
\hline$n_{\max }$ & $(0.2,0.2)$ & $(0.5,0.5)$ & $(0.6,0.8)$ & $(0.8,0.3)$ \\
\hline \multicolumn{5}{|c|}{ Bi $\gamma=0.1$ and $\beta=1$} \\
\hline 5 & 0.0890434 & 0.089842 & 0.0888428 & 0.0894994 \\
\hline
\end{tabular}




\begin{tabular}{|c|c|c|c|c|}
\hline 10 & 0.0890504 & 0.0899103 & 0.0888709 & 0.0895157 \\
\hline 20 & 0.0890448 & 0.089896 & 0.0888697 & 0.0895100 \\
\hline 40 & .0890452 & 0.0898979 & 0.0888695 & 0.0895108 \\
\hline 60 & 0.0890453 & 0.0898981 & 0.0888695 & 0.0895109 \\
\hline 80 & 0.0890453 & 0.0898982 & 0.0888695 & 0.0895109 \\
\hline 100 & 0.0890453 & 0.0898982 & 0.0888695 & 0.0895109 \\
\hline $\begin{array}{c}\text { CITT-DT } \\
\text { Converged }\end{array}$ & 0.0890453 & 0.0898982 & 0.0888695 & 0.0895109 \\
\hline \multicolumn{5}{|c|}{$\operatorname{Bi} \gamma=0.01$ and $\beta=1$} \\
\hline 5 & 0.890044 & 0.890843 & 0.889842 & 0.890503 \\
\hline 10 & 0.890051 & 0.890912 & 0.889870 & 0.890519 \\
\hline 20 & 0.890045 & 0.890897 & 0.889868 & 0.890513 \\
\hline 40 & 0.890045 & 0.890899 & 0.889868 & 0.890514 \\
\hline 60 & 0.890045 & 0.890899 & 0.889868 & 0.890514 \\
\hline 80 & 0.890045 & 0.890899 & 0.889868 & 0.890514 \\
\hline 100 & 0.890045 & 0.890899 & 0.889868 & 0.890514 \\
\hline $\begin{array}{c}\text { CITT-DT } \\
\text { Converged }\end{array}$ & 0.890045 & 0.890899 & 0.889868 & 0.890514 \\
\hline \multicolumn{5}{|c|}{$\operatorname{Bi} \gamma=0.1$ and $\beta=0.8$} \\
\hline 5 & 0.0891571 & 0.090045 & 0.0886542 & 0.0896702 \\
\hline 10 & 0.0891686 & 0.0901443 & 0.0886977 & 0.089693 \\
\hline 20 & 0.0891600 & 0.0901223 & 0.0886959 & 0.0896842 \\
\hline 40 & 0.0891607 & 0.0901253 & 0.0886956 & 0.0896854 \\
\hline 60 & 0.0891608 & 0.0901256 & 0.0886955 & 0.0896856 \\
\hline 80 & 0.0891609 & 0.0901257 & 0.0886955 & 0.0896856 \\
\hline 100 & 0.0891609 & 0.0901257 & 0.0886955 & 0.0896856 \\
\hline $\begin{array}{c}\text { CITT-DT } \\
\text { Converged }\end{array}$ & 0.0891609 & 0.0901257 & 0.0886955 & 0.0896856 \\
\hline \multicolumn{5}{|c|}{$\operatorname{Bi} \gamma=0.01$ and $\beta=0.8$} \\
\hline 5 & 0.890160 & 0.891047 & 0.88965 & 0.890676 \\
\hline 10 & 0.890171 & 0.891146 & 0.889693 & 0.890698 \\
\hline 20 & 0.890163 & 0.891124 & 0.889692 & 0.890690 \\
\hline 40 & 0.890164 & 0.891127 & 0.889691 & 0.890691 \\
\hline 60 & 0.890164 & 0.891128 & 0.889691 & 0.890691 \\
\hline 80 & 0.890164 & 0.891128 & 0.889691 & 0.890691 \\
\hline 100 & 0.890164 & 0.891128 & 0.889691 & 0.890691 \\
\hline $\begin{array}{c}\text { CITT-DT } \\
\text { Converged } \\
\end{array}$ & 0.890164 & 0.891128 & 0.889691 & 0.890691 \\
\hline
\end{tabular}

Table 3 shows that the CITT-ST approach converges faster than the CITT-DT. For Bi $=0.01$ and $\beta=0.8$ the summation of 40 terms obtains the same value as CITT-DT, which required 500 terms for the convergence at position $(0.2,0.2)$. For Bi $\gamma$ $=0.1$ and $\beta=1$ at position $(0.5,0.5)$ required 60 terms using CITT-ST against 1000 for double.

The results show that CITT-ST is more efficient to obtain the solution for heat conduction in Solid State Electronics even though it has a more complex formulation.

\section{CONCLUSIONS}

This paper presented the comparison of the solution by Classical Integral Transform Technique (CITT) of a solid-state device with heat generation and external convection by single transformation (CITT-ST) and double transformation (CITT-DT) approaches. It was presented an SSE with a symmetrical layout of components but with different heat generation intensities in each component. It was shown the convergence for different values of $\mathrm{Bi}$ and in different positions of the domain. Both approaches were compared by its solution methodology and a convergence analysis was performed.

The formulation of the problem by CITT-DT presents a more compact and simple solution, resulting in an algebraic system of equations. On the other hand, the CITT-ST approach presents a complex and cumbersome analytical solution. The convergence analysis, however, showed that CITTST has a greater performance than the CITT-DT for the case with multiple heat generation over the chip. CITT-ST required very few terms and obtained a fully converged six-digits solution.

Finally, the integral transform technique has shown to be a good alternative method for this kind of problem and the CITT-ST has performed better for this case than CITT-DT.

\section{ACKNOWLEDGEMENTS}

The authors would like to acknowledge the financial support provided by Universidade do Estado do Rio de Janeiro and FAPERJ.

\section{REFERENCES}

Abdelmlek, K. B., Araoud, Z., Ghnay, R., Abderrazak, K., Charrada, K., and Zissis, G., 2016, Effect of Thermal Conduction Path Deficiency on Thermal Properties of LEDs Package, Applied Thermal Engineering, Vol. 102, pp. 251-260.

Bar-Cohen, A., and Wang, P., 2009, On-chip Hot Spot Remediation with Miniaturized Thermoelectric Coolers, Microgravity Science and Technology, Vol. 21, Supplement 1, pp. 351-359.

Braga Junior, N. R., and Sphaier, L. A., 2014, Effective Thermal Conductivity of Composite Materials Using the Integral Transform Technique, in: 15th Brazilian Congress of Thermal Sciences and Engineering, ENCIT.

Chalhub, D. J. N. M., Sphaier, L. A., and Alves, L. S. de B., 2014, Integral Transform Analysis of Poisson Problems that Occur in Discrete Solutions of the Incompressible Navier-Stokes Equations, Journal of Physics: Conference Series, Vol. 547, Conference 1, pp. 1-10.

Chelikowsky, J. R., and Franciosi, A., 1991, Electronic Materials: a New Era in Materials Science, Springer-Verlag Berlin Heidelberg.

Cotta, R. M., 1993, Integral Transforms in Computational Heat and Fluid Flow, 1st Edition, CRC Press.

Dantas, L. B., 1996, Estudo da Transferência de Calor por Encapsulamento Plástico Usando a Técnica da Transformada Integral Generalizada, Master Thesis, UFRJ, Rio de Janeiro, RJ. (in portuguese)

Hahn, D. W., and Özisik, M. N., 2012, Heat Conduction, 3rd Edition, John Willy \& Sons, Inc.

Iyengar, M., and Schmidt, R., 2006, Analytical Modeling for Prediction of Hot Spot Chip Junction Temperature for Electronics Cooling Applications, 
in: Thermal and Thermomechanical Proceedings 10th Intersociety Conference on Phenomena in Electronics Systems, ITHERM'06.

Peterson, G. P., and Ortega, A., 1994, Thermal Control of Electronic Equipment and Devices, Advances in Heat Transfer, Vol. 20, pp. 181-314.

Rinaldi, N., 2000, Thermal Analysis of SolidBase Devices and Circuits: an Analytical Approach, Solid-State Electronics, Vol. 44, pp. 1789-1798.

Silva, L. C. D., Chalhub, D. J. N. M., Calil, A. L. O., and de Moura, R. S., 2017, Semi-Analytical Solution of the Heat Conduction in a Plate with Heat Generation, Engenharia Térmica (Thermal Engineering), Vol. 16, No. 1, pp. 58-65.

Sphaier, L., and Cotta, R., 2000, Integral Transform Analysis of Multidimensional Eigenvalue Problems within Irregular Domains, Numerical Heat Transfer Part B, Vol. 38, pp. 157-175.

Xiao, C., He, H., Li, J., Cao, S., and Zhu, W., 2017, An effective and Efficient Numerical Method for Thermal Management in 3D Stacked Integrated Circuits, Applied Thermal Engineering, Vol. 121, pp. 200-209. 